\title{
Peer-to-Peer Session Initiation Protocol in Highly Volatile Environments
}

\author{
Adetola Oredope ${ }^{1}$, Antonio Liotta ${ }^{1}$, Ivan Roper ${ }^{2}$ and Jason Morphett ${ }^{2}$ \\ ${ }^{1}$ University of Essex, Wivenhoe Park, Colchester, CO4 3SQ, United Kingdom \\ ${ }^{2}$ British Telecommunications (BT), Adastral Park, Martlesham Heath, IP5 3RE, United Kingdom \\ \{aoredo; aliotta\}@essex.ac.uk,\{ivan.roper;jason.morphett\}@bt.com
}

\begin{abstract}
The Session Initiation Protocol (SIP) is an IETF standardised protocol for multimedia signalling and communication over the internet. SIP has been used in many deployments in client-server architecture. However, this configuration potentially possesses various scalability and redundancy limitations because its architecture relies on various centralised components. To overcome some of these limitations, there has been various proposed architectures for using SIP in a fully decentralised manner with minimal or no centralised authorities. One of these proposed architectures is known as Peer-to-Peer SIP (P2P-SIP), which uses structured $p 2 p$ overlay networks to lookup and locate SIP resources over the Internet. In this paper we investigate P2P-SIP in a broader perspective beyond the Internet, focusing on the effects of mobility on this approach especially in highly volatile environments in which the underlying $p 2 p$ overlay never fully converges or stabilises. Our experimental results shows that P2P-SIP systems using CHORD deployed in these environments are highly scalable. But more interestingly, we also show that as the number of peers in the system increases, the signalling overheads per peer in the overlay tends to decrease.
\end{abstract}

\section{Introduction}

Converged networks in the context of this paper refer to the deployment of rich services, merging both fixed and mobile networks. This allows for better Quality of Service (QoS), charging mechanisms and integration of services as compared to conventional fixed or mobile networks individually [1]. These services include Voice over Internet Protocol (VoIP), Push-to-Talk, Click-to-Dial, online gaming or an integration of these services. Most of these multimedia services are based on the Session Initiation protocol (SIP) [2] which is an IETF standardised protocol for session management and control over the Internet. SIP allows clients to invite other clients into a session; it then negotiates control information, terminal capabilities and media channels to be used in the session, then the clients can be connected directly via the Internet Protocol (IP). Therefore, SIP is mainly used to locate session participants in multimedia communications [3]. This is invariably achieved by using centralised SIP servers for storing, routing and manipulating signalling information.

One of the limitations of this centralised approach is scalability; more servers and resources need to be added to the SIP network as the number of clients increases, causing an increase in complexity [4]. Another such limitation of centralised SIP servers is redundancy, especially in times of major disasters in which the centralised infrastructure could potentially act as a single point of failure [5]. Most importantly, especially in relation to this paper, the potential for restricted flexibility and additional costs involved in the deployment of SIP based applications and services in smaller and adhoc environments such as end-users homes, small and medium enterprises, conferences, temporary posts and outdoor/field operations. These applications currently require the deployment of servers and additional support components in order to benefit from the advantages of SIP.

To overcome these centralised limitations of SIP, there has been a variety of approaches in using a decentralised or peer-to-peer ( $\mathrm{P} 2 \mathrm{P})$ approach such as: SOSIMPLE [6]; P2P Internet telephony using SIP [4]; SIPDHT [7]; NimX [8]; and P2P-SIP [9] to mention a few. Most of these approaches aim to use SIP over a highly scalable distributed overlay network allowing all the SIP resource lookups and locations to be carried by the underlying distributed overlay network. These approaches allow the SIP network to scale to a high number of users with limited centralisation as long as the overlay network fully converges or stabilises. Theoretically high efficiency levels of scalability of overlay networks have already been demonstrated in existing distributed and P2P file sharing networks [10]. 
In this paper however, we investigate further the extent of efficiencies in both: (i) scalability and; (ii) redundancy properties of P2P-SIP when used with CHORD [11]. In particular, investigating these properties in an environment in which the underlying P2P overlay network never fully stabilises or converges such as in highly volatile environments. The instability could be as a result of peers constantly leaving and joining the P2P-SIP overlay due to various mobility factors such as handovers, fluctuating connections and loss of wireless signals. This can lead to a varying level of churn in the system of which we are particularly interested in the effects on the scalability and redundancy properties on P2P-SIP overlays.

Our aim in this paper is to carry out various scalability and redundancy tests on a P2P-SIP system under highly volatile conditions with less emphasis on the underlying P2P overlay technology. Although we are aware the underlying overlay may affect the overall performance, we have chosen to study and analyse P2P-SIP at a higher level. Based on this, we used Telecom Italia's implementation of P2P-SIP known as SIPDHT [7], which is based on the integration of SIP and a highly scalable and redundant structured $\mathrm{p} 2 \mathrm{p}$ overlay network known as CHORD The results from our experimental studies using this implementation configuration shows that P2P-SIP systems in mobile environments are highly scalable even with high numbers of peers, in accordance with the accompanying theoretical proposals and propositions of the underlying systems. But more interestingly, we also show that as the number of peers in the system increases, the signalling overheads per peer in the overlay tends to decrease which in returns significantly reduces the overall bandwidth consumed by the P2PSIP system as a whole. Our results also show how the performance of the system (i.e. amount of bandwidth consumed) is affected by the varying levels of churn and the distribution of keys in the hash tables in the P2P-SIP system.

Section 2 provides a brief overview on the current research and commercial developments in P2P-SIP and also on the current status of the P2P-SIP IETF working group. Section 3, describes our test bed, experiments and results. In section 4 we discuss the outcomes of the experiments and recommendations. Finally in section 5 we provide conclusions and directions for future work.

\section{Related Work}

As described earlier, P2P-SIP implementations rely on the underlying P2P network in order locate SIP resources with little or no centralised systems. At the moment, there are various commercial and research interests in the development of P2P-SIP services not as a total replacement for the existing SIP system infrastructure but as an additional service or extension to further enhance the functionality of conventional SIP. Although, most of the research efforts in developing P2P-SIP are currently concentrated in the P2P-SIP IETF working group [9], there were two main proposals that served as the foundation of the research and commercial deployments.

The first proposal is the Self Organising SIP for Instant Messaging and Leveraging Extension (SOSIMPLE) [6] developed at The College of William and Mary. This platform reuses technologies such as VoIP clients, network infrastructures, and open source protocol stacks. Their implementation is based on the integration of SIP [2] and SIMPLE [12] messages with P2P algorithms such as CHORD [11] replacing the basic SIP server functionality. Just like other P2P-SIP implementations, it aims to avoid the traditional centralized architecture found in SIP and replace servers with a peer-to-peer overlay network, allowing nodes to act as User Agents (UA) and also perform key functions that would conventionally belong to SIP servers i.e. proxy and registration. This approach allows for conventional SIP messages like REGISTER and INVITE to be used. Instead of proposing a new protocol, SOSIMPLE redefines the header of SIP messages to allow SIP messages to carry P2P traffic in compliance to the IETF standards for SIP. This also allows for backward compatibility and interoperability with other existing implementations.

The second approach was proposed by Columbia University and is known as the P2P Internet Telephony Using SIP [4], which is based on a hierarchical approach using conventional SIP and different forms of Distribute Hash Tables (DHT). Compared to the SOSIMPLE implementation, this does not assume that all nodes have enough resources to implement a DHT. This approach selects super nodes based on high capacity of resources (bandwidth, CPU, memory) and availability (uptime, public address) allowing ordinary nodes to connect to the overlay network via the super nodes. The use of super nodes has been known to be exploited and used in commercial implementations like Skype [13] (for P2P VoIP and multimedia communications) and Joost [14] (for multimedia streaming).

In order to consolidate the various implementations of P2P-SIP and also allow for interoperability between these systems, the P2P-SIP IETF working group was setup. It defines P2P-SIP Overlays as a collection of 
P2P-SIP peers in which the P2P network provides the location server functionality and also provides the transport function for the exchange of SIP messages between nodes [9]. The working group also recommends that P2P-SIP runs a distributed database algorithm which allows data to be stored and retrieved from different nodes in the overlay. Each peer can provide individual service and the overlay can also provide a collective service to all peers in the overlay. The IETF working group has also defined P2P-SIP entities which are meant hold only the client role, i.e. they do not have to contribute any service to the overlay network. These should be considered as a specialisation (or subset) of ordinary peers. P2P-SIP peers and clients can also be coupled with conventional SIP functionalities, allowing them to be functional in both conventional SIP and P2P-SIP networks. Currently various internet drafts have been proposed to the IETF in order to justify the standardization of the P2P-SIP protocol but as yet none of these drafts has been standardized. More information about the current drafts can be found on the IETF P2P-SIP WG website [9] .

In this paper we focus on carrying out a performance analysis on the early implementations of the IETF P2P-SIP protocols especially in the context of highly volatile environments in which the underlying P2P network never seems to converge due to peers continually joining and leaving the overlay. The reason for doing this is to best simulate what we believe a 'practical' network doing, i.e. taking theoretical design into a pseudo-practical simulation to assess behaviour. Hitherto, we are not aware of other publicly available research in this area and we hope that these initial results will provide more insight into the scalability and redundancy properties of P2P-SIP in mobile environments and also provide a foundational building block in the development of services in mobile environments.

\section{Experimental Tests and Analysis}

To further investigate the characteristics and behaviour of P2P-SIP in mobile environments we carried out various tests and analyses that allowed us to monitor, capture and evaluate various parameters such as the amount of packets exchanged between peers, the amount of signalling generated, and the amount bandwidth consumed by individual peers participating in P2P-SIP overlay as a whole. In this section we describe the implementation of the test bed which allowed us to run a number of peers in a controlled environment. We also present the results and analysis of the simulations.

\subsection{Test Bed Implementation}

There are various implementations of P2P-SIP as described in Section 1 but only a few of these implementations are open-source and publicly available. In our test bed we used Telecom Italia's SIPDHT [7] implementation because it is currently one of the most mature open-source implementations of P2P-SIP available. Furthermore, SIPDHT uses CHORD [11] as the underlying structured P2P algorithm and Nokia's open source Sofia SIP stack [15] for SIP signalling. Even though SIPDHT is no longer supported by its developers, due to the release of its second version, SIPDHT2 which is built on Content Addressable Network (CAN) [16], we found the first version to be more stable, easily extendable to accommodate some modifications we made to it in order to scale beyond the default.



Table 1: Test Bed Application Pseudo Code

In order to deploy a large amount of nodes easily and across multiple infrastructures we developed a parallelised algorithm as shown in Table 1. The algorithm allows for a rapid generation of peers based on the available resources on the infrastructure. The main advantage of this approach is that each peer generated is allocated a certain amount of resources based on the parameters set in the algorithm. These parameters allow node properties for both mobile and fixed nodes to be simulated. Another advantage of this 
algorithm is that it allows various tests and applications to be run on a large scale and at same time allowing various monitoring data to be captured. We used tcdump [17] for capturing and monitoring events on the test bed because it provides an avenue to extract rich forms of data while analysing the captured information.

The algorithm described was implemented and deployed over a high performance computing infrastructure made up 12 Intel Quadcore high end nodes with each node having an individual $2 \mathrm{~GB}$ of RAM, 250GB of disk space and then an external attached RAID storage of 6TB using Network File System. We chose to use such a high end infrastructure because it will enable our test bed run applications that can span to over a millions nodes. Parallel communication between individual nodes is based on the Sun Grid Engine (SGE) [18] which allowed to us to provide a platform for distributed and parallel processing. In order to validate our test bed over 120 tests were carried out ranging from interconnectivity tests to inter process communications.

\subsection{P2P-SIP in Mobile Environments}

Once the test bed was setup and validated, we carried out various tests to simulate the behaviour of P2P-SIP in mobile and high volatile environments investigating how the overlay behaves and reacts to peer joins and leaves which is a common issue in mobile networks. We carried two tests in which the first test is based on nodes just leaving the system and then allowing the system to fully converge. However, in the second test peers continually joined and left the system whilst never allowing the overlay to fully converge.

Test 1: $P 2 P-S I P$ in an environment in which peers constantly leave the overlay over a constant period of time allowing the overlay to converge.

Aim: The aim of this test is to study the characteristics of the $\mathrm{p} 2 \mathrm{p}$-sip overlays in a mobile environment during periods in which nodes continually leave the system over a constant time. An increasing number of nodes were forced to leave the overlay within ten seconds.

Setup: The testbed was set up to generate nodes sets ranging between 10 nodes to 200 nodes. For each node set (for example an overlay with 40 nodes), an incremental amount of nodes were killed over 10 seconds (i.e one node was killed after 10 seconds, then two nodes over 10 ten seconds and so on until 10 nodes were killed over 10 seconds.). Each scenario was simulated 10 times in order to minimise errors and the average data over each scenario was collected

Results: The stacked graph shown in Figure 1 describes the effect of an increasing number of peers leaving the overlay over a constant period of ten seconds. It shows a consistent behaviour in the amount of packets generated over 10 seconds. This behaviour is similar to the characteristics of the underlying $p 2 p$ protocol CHORD.

Figure 1 also shows that the amount of signalling packets generated tends to scale favourably even with higher numbers of peers i.e generating a lower amount of packets as the number of peers leaving the system increases. This we have discovered is heavily dependent on the number of entries in the node finger tables. A key finding from this test was that with a higher number of peers in the overlay; a lesser fraction of the overlay is actually updated.

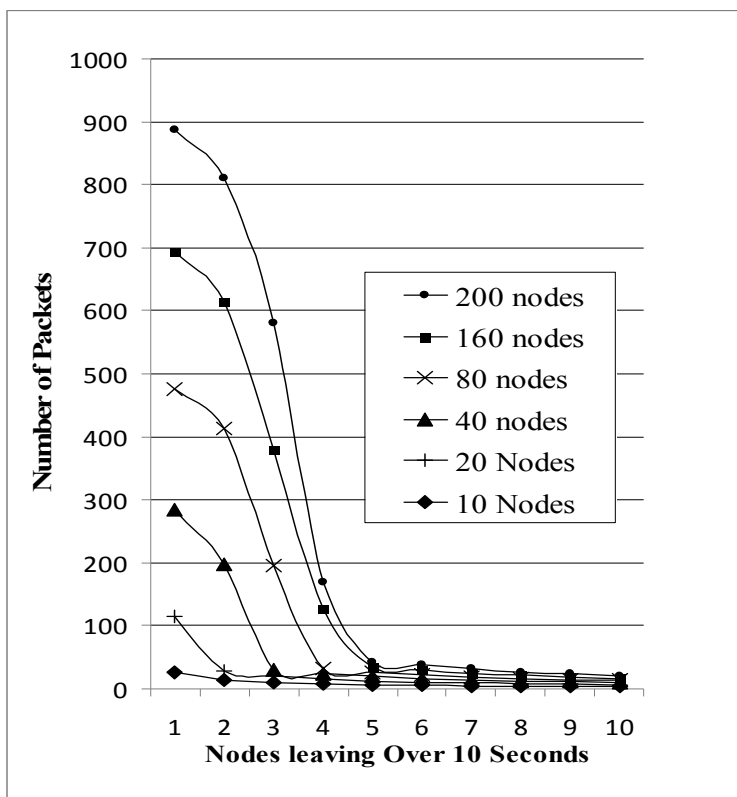

\section{Figure 1: The Effects of Nodes (Peers) Leaving the Overlay}

This behaviour was consistent across all tests and reveals a scalability trait in P2P-SIP. Also from the stacked graph in Figure 1, we can show that that for higher numbers of peers in the system, for example, at 200 peers, when a single peer leaves the overlay more signalling is generated when compared with a higher number (i.e 10 peers) leaving the same system. Although this may appear detrimental with a lower number of peers leaving the overlay, as the number of 
peers leaving the system increases the signalling is greatly reduced

Test 2: $P 2 P-S I P$ in an environment in which peers constantly join and leave the overlay over a constant period of time without allowing the overlay to converge

Aim: The aim of these tests is to study and understand what happens in the overlay when continuous peer join and leaves never allow the system to converge (stabilise).

Setup: In order to emulate this type of environment, P2P-SIP overlays were again generated using 10 and 200 peers. Once the overlay was fully converged, varying churn rates were introduced into the system. The peers in the overlay were constantly forced to leave and join the overlay over a fixed period of 300 seconds. For example for a churn rate of $40 \%, 20 \%$ of the peers are continually forced to leave the overlay while another $20 \%$ are forced to join at an incremental interval of 30 s until a total time of 300 seconds is reached. The time specified for each scenario was fixed at 300 seconds throughout all the experiments. All experiments for each scenario were carried out 10 times to minimise any effects of errors.

Results: The results for these experiments are shown in Figures 2, 3 and 4. Based on the results collected for the overall system during the tests, Figure 2 shows a consistent pattern between of all the values of the churn rates used. The maximum amount of bandwidth consumed tends to peak at a particular point and then decrease rapidly. We believe this attribute demonstrated shows that at higher churn rates the bandwidth consumed by the system is lower. Although logically, more signalling packets are expected to be generated with higher numbers of peers, from Figure 2 we can see that at $10 \%$ churn rate for 20 nodes causes 2122 signalling packets which in returns consumes a bandwidth of $40.42 \mathrm{Kbits} / \mathrm{s}$ compared to 200 peers which generated 4093 packets using a bandwidth of $78.78 \mathrm{Kbits} / \mathrm{s}$.

Even though the amount of packets generated and bandwidth consumed in 200 peers is far higher than that of the 10 peers, the graph in Figure 2 shows that the relative bandwidth is in fact, dropping. To help understand the behaviour and the drop on the bandwidth, Figure 3 shows the amount of signalling generated by each peer in the overlay. We plotted the graph of the bandwidth consumed by individual peers which shows that each peer in the overlay actually consumes less bandwidth as the number of nodes increases (Figure 3).



Figure 2: Average Bandwidth in the Overlay

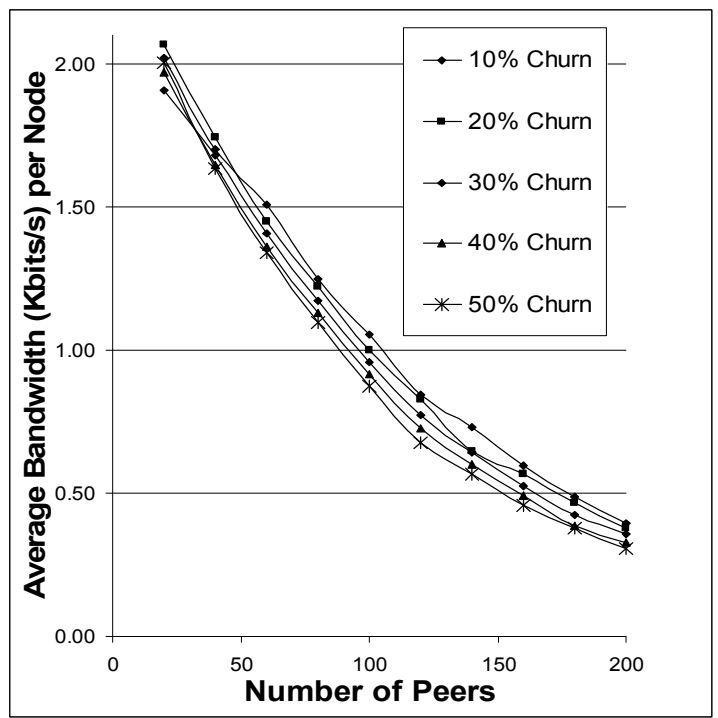

Figure 3: Average Bandwidth Per Peer

Moreover from the graph in Figure 3, it can be seen that on individual nodes the amount of bandwidth generated reduces as the churn increases and this could be due to various factors. One of these factors is based on the finger tables. Our supposition is that with a higher number of peers in the overlay, only a small fraction of the ring is affected by any join or leave updates which in turn leads to a reduced level of signalling. Another factor that could be responsible for this attribute is based on the key space or identifier chord circle [11] in which each space in the ring 
allocated a fixed key, so when a node leaves the ring and the same node or a new node returns, it returns to the same space in the identifier circle or key space previously vacated. In this situation, the peer's neighbours may not have noticed that the peer had previously left the overlay for a while and therefore, no extra overhead signalling is generated to make up for this disappearance.

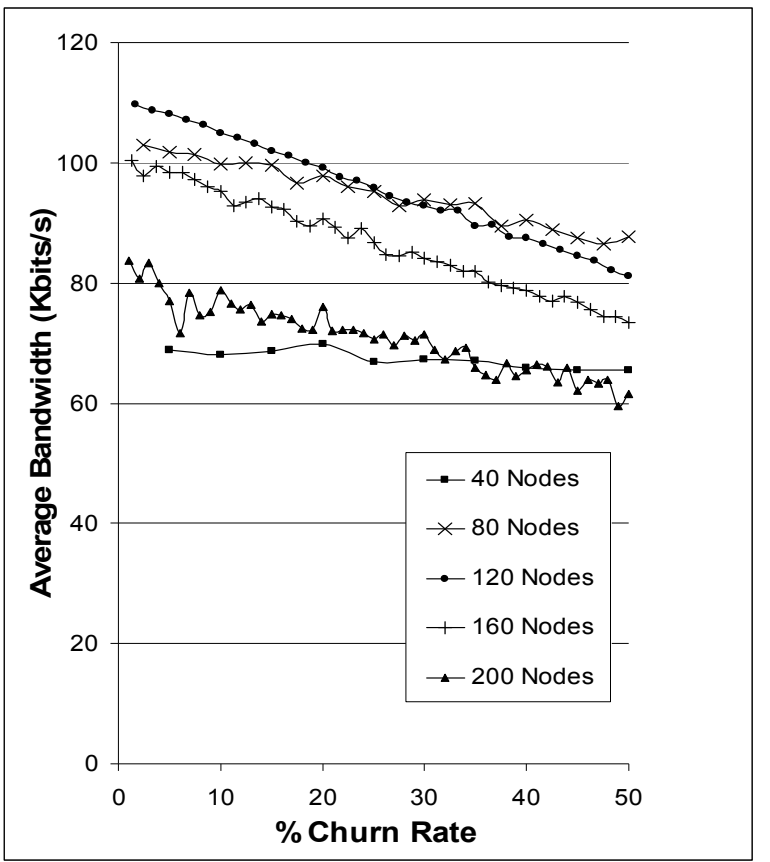

Figure 4: Average Bandwidth in terms of Churn Rate

In order to further confirm these attributes demonstrated by the P2P-SIP overlay, we plotted of the average bandwidth in the overlay versus the churn rates, this is shown in Figure 4. Paradoxically perhaps, but this graph again confirms that the amount of bandwidth generated is actually reduced as churn rate increases.

\section{Discussion and Recommendations}

Based on the results and graphs in Section 3, we can confirm that under this configuration of P2P-SIP using SIPDHT (with CHORD), the protocol appears to scale well in mobile and volatile environments. Although there are higher amounts of signalling that are generated versus a centralised system, we believe the network operator / service provider could calculate the cost of these signalling overheads and compare them with the cost of running and maintaining centralised resources for conventional SIP. We also believe the network operator / service provider can take advantages of P2P-SIP systems and provide a Web 2.0 array of services in which different peers develop new services and even host these services.

The P2P-SIP architecture is still not fully matured and various issues still need to be considered such as security (which peers can be trusted?) etc. However, these are issues outside the scope of this paper as we have focussed specifically on understanding it behaviour under stressful conditions.

Overall, our experimental results and analysis show that in highly volatile environments the system as described performs well. In particular, the amount of signalling generated tends to increase initially and then reduces sharply, to a point whereby the system stabilises. This point of stablisation appears to be at a constant bandwidth that can be predicted. This prediction will be the subject of further work.

\section{Conclusion}

P2P-SIP allows multimedia sessions to be generated, modified or terminated in a distributed approach with little or no centralised authorities. In this paper we have outlined ongoing research and commercial interests in P2P-SIP also looking at its standardisations process at the IETF. We carried out various experiments to study the performance of P2PSIP in a highly volatile environment since SIP is used for session signalling in converged networks which provides access to both fixed and mobile end-users. Our results show that in the presence of mobile and volatile factors, P2P-SIP with SIPDHT tends to scale quite well even though it generates more signalling than conventional SIP. The overall - per node consumed bandwidths also tended towards a download slope allowing the overlay to scale quite well.

Finally we believe P2P-SIP will provide various advantages, such as in the development of new services and the use of rich multimedia applications in smaller and adhoc environments like homes, small and medium enterprises, conferences, temporary posts and outdoor/field operations

\section{References}

[1 ] G. Camarillo and M.A. Garcâia-Martâin, The 3G IP multimedia subsystem (IMS) : merging the internet and the cellular worlds, Chichester, West Sussex ; Hoboken, NJ: J. Wiley, 2006.

[2 ] J. Rosenberg et al., "SIP: Session Initiation Protocol," RFC 3261 - Internet Engineering Task Force, Jun. 2002.

[3 ] G. Camarillo, SIP demystified, New York: McGrawHill, 2002. 
[4 ] K. Singh and H. Schulzrinne, "Peer-to-peer internet telephony using SIP," Proceedings of the international workshop on Network and operating systems support for digital audio and video, Stevenson, Washington, USA : ACM Press, 2005, pp. 63-68 .

[5 ] D.A. Bryan et al., "SOSIMPLE: A Serverless, Standards-based, P2P SIP Communication System SOSIMPLE: A Serverless, Standards-based, P2P SIP Communication System," Advanced Architectures and Algorithms for Internet Delivery and Applications, 2005. AAA-IDEA 2005. First International Workshop on, 2005, pp. 42-49.

[6 ] D.A. Bryan, B.B. Lowekamp, and C. Jennings, "SOSIMPLE: A Serverless, Standards-based, P2P SIPCommunication System," Advanced Algorithms and Architecture for the Internet, Jun. 2005.

[7 ] Telcom-Italia, "SIPDHT," http://sipdht.sourceforge.net/.

[8 ] N. Nimcat, "nimX - P2P-SIP call processing software," http://www.nimcatnetworks.com/.

[9 ]"Peer-to-Peer Session Initiation Protocol (P2P-SIP)," http://tools.ietf.org/wg/p2psip/.

[10] L. Eng Keong et al., "A survey and comparison of peerto-peer overlay network schemes," Communications Surveys \& Tutorials, IEEE, vol. 7, 2005, pp. 72-93.

[11] I. Stoica et al., "Chord: a scalable peer-to-peer lookup protocol for Internet applications," Networking, IEEE/ACM Transactions on, vol. 11, 2003, pp. 17-32.

[12] B. Campbell et al., "Session Initiation Protocol (SIP) Extension for Instant Messaging," RFC 3428 - Internet Engineering Task Force, Dec. 2002.

[13] S.A. Baset and H. Schulzrinne, "An Analysis of the Skype Peer-to-Peer Internet Telephony Protocol," http://arxiv.org/ftp/cs/papers/0412/0412017.pdf, Sep. 2004.

[14] Joost, http://www.joost.com/.

[15] Nokia, "Sofia SIP," http://opensource.nokia.com/projects/sofia-sip/.

[16] R. Sylvia et al., "A scalable content-addressable network," 2001.

[17]“TCPDUMP - Network Sniffer," p. http://www.tcpdump.org/.

[18] Sun-Microsystems, "Sun Grid Engine," http://gridengine.sunsource.net/. 